\title{
Engel-Brewer Electron Correlation Model: A Critical Discussion and Revision of Concepts
}

\author{
S. Raju, E. Mohandas and V. S. Raghunathan \\ Physical Metallurgy Section, Metallurgy Division, Indira Gandhi Centre for Atomic Research, \\ Kalpakkam 603 102, India
}

\begin{abstract}
A critical review of the Engel-Brewer theory of metallic bonding is presented with an emphasis on its inapplicability to transition metals. Taking recourse to pseudopotential arguments for nontransition metals and to tight binding model for d-transition metals, the cohesive and crystal structure trends among metals across the periodic table is briefly discussed. It is clear that a direct inclusion of d-electron counts is absolutely necessary to correlate meaningfully the observed structural trend with the valence electron configuration. In view of this limitation, the classical Brewer's scheme is revised in such a manner that some of the anomalies encountered in the case of transition metals are removed. A discussion of the philosophical basis of Brewer's model is presented.
\end{abstract}

(Received May 8, 1995)

Keywords: Engel-Brewer model, structural stability, electron to atom ratio, transition metals

\section{Introduction}

The Engel-Brewer electron correlation model (EBECM) or Engel-Brewer rules (EBR), as they have to be called appropriately remain till date one of the controversial areas in the theory of alloying ${ }^{(1)(2)}$. These rules proposed first by Engel ${ }^{(3)}$ on empirical grounds, elaborated and popularised subsequently by Brewer in a series of publications ${ }^{(4)}$ are basically correlations relating the valence electron concentrations of metals and alloys as they occur in the condensed state with their crystal structure. Although empirical in essence, its applications to basic problems of structural metallurgy is rather extensive and is growing unabated ${ }^{(4)-(7)}$. A possible origin of EBR may be traced to the classical valence-bond theory in chemistry ${ }^{(8)}$, but nevertheless has no undisputed base in solid state physics ${ }^{(1)(3)(9)-(11)}$. Stating briefly at this point, a chemical interpretation of EBR invokes the presence of well defined $\mathrm{s}$ and $\mathrm{p}$ orbitals in the condensed state, a proposition inconsistent with the basic tenets of a band theoretical approach ${ }^{(1)}$. Not surprisingly therefore, the EBR have drawn adverse criticism from physicists. For details in this respect we refer to the overview of Brewer and the consequent panel discussions that it had drawn in the 1967 Batelle conference on alloy phase stability ${ }^{(11)}$. The Engel-Brewer viewpoints as enunciated by Brewer in 1967 have remained virtually unchanged as of date, while that of solid state physicist have undergone revision and extension in the mean time ${ }^{(12)(13)}$. In view of this it is appropriate to reevaluate the physical reasons for the success or lack of it of the Engel-Brewer scheme in the light of the advances that have been made from time to time in solid state theory. It is interesting to recall here the case of another electron concentration based model, namely the Hume-Rothery rules, which in turn has prompted many a theoretical attempt to unravel the underlying physical picture. Even as the modern theory of alloying is making successful compromises between the classical chemical and modern physical viewpoints ${ }^{(14)}$, it is rather instructive at this stage to launch a fresh inspection of the fundamental postulates of EBECM, with a view to extend it in accordance with the prevailing physical viewpoints on the crystal structure trends. This is the objective of this paper.

The subject matter of this paper is organised in the following manner. In section two a brief summary of EBECM is presented with an emphasis on the enunciation of its basic aspects. The limitations are clearly listed and whereby it is pointed out that the direct inclusion of $\mathrm{d}$ electrons is mandatory to extend the application to transition metals and their alloys. In Section III, we digress a little to discuss in general certain issues related to crystal structure trends in transition and non transition metals. We discuss here the diverse views of metallic binding rather briefly. In Section IV we arrive at an extended electron configuration scheme for metals by using well established principles of metallic cohesion as discussed in Section III. A discussion on the philosophical nature of the problem is presented in the concluding section.

\section{Engel-Brewer Model: A Brief Description}

The Engel-Brewer scheme assumes that atoms of an element may first be excited to promote their valence electrons to a different bonding state and subsequently condensing them to form a metal whose crystal structure is decided solely by the number of unpaired $s$ and $p$ electrons in the excited state. The promotion to the excited state is effected only if the promotion energy cost is offset by the enhanced metallic binding made possible by optimising the number of unpaired electrons. Further the ex- 
citation from a completely filled inert gas like shell is not normally permitted.

For non transition elements there seems to be no serious difficulties in considering the $s$ and $p$ orbitals of atoms as forming the metallic valence state ${ }^{(14)(15)}$; however some complications arise in the case of immediate post transition elements. For the transition metals however, the Engel-Brewer scheme postulates that d electrons do take place in bonding, although indirectly by allowing for $\mathrm{d}-\mathrm{s} / \mathrm{p}$ mixing or hybridization. The crucial point is

Table 1 Electronic configurations, promotion energies and crystal structures of elements.

\begin{tabular}{|c|c|c|c|c|c|c|c|c|c|}
\hline Elements & $\begin{array}{c}\text { Electron } \\
\text { configuration }\end{array}$ & $\begin{array}{c}\text { Promotion } \\
\text { energy } \\
(\mathrm{kJ} / \mathrm{mol})\end{array}$ & $\begin{array}{l}\text { Crystal } \\
\text { structure } \\
\text { as per } \\
\text { EBECM }\end{array}$ & Remarks & Elements & $\begin{array}{c}\text { Electron } \\
\text { configuration }\end{array}$ & $\begin{array}{c}\text { Promotion } \\
\text { energy } \\
(\mathrm{kJ} / \mathrm{mol})\end{array}$ & $\begin{array}{l}\text { Crystal } \\
\text { structure } \\
\text { as per } \\
\text { EBECM }\end{array}$ & Remarks \\
\hline $\mathrm{Na}$ & $\mathrm{S}$ & 0 & bcc & & & $d(3) s$ & 59 & bcc & \\
\hline \multirow[t]{2}{*}{$\mathrm{Mg}$} & $s(2)$ & 0 & - & & & $d(2) s p$ & 175 & hcp & \\
\hline & $\mathrm{sp}$ & 263.6 & hep & & $\mathrm{Nb}$ & $d(4) s$ & 0 & bcc & \\
\hline \multirow[t]{2}{*}{$\mathrm{Al}$} & $s(2) p$ & 0 & - & & & $d(3) s p$ & 200 & - & \\
\hline & $\mathrm{sp}(2)$ & 347.3 & fcc & & Mo & $\mathrm{d}(5) \mathrm{s}$ & 0 & bcc & \\
\hline $\mathrm{K}$ & $\mathrm{s}$ & 0 & bcc & & $\mathrm{Tc}$ & $\mathrm{d}(5) \mathrm{s}(2)$ & 0 & - & \\
\hline \multirow[t]{3}{*}{$\mathrm{Ca}$} & $s(2)$ & 0 & - & & & $d(6) s$ & 29 & - & \\
\hline & $\mathrm{sp}$ & 180 & - & $*$ & & $d(5) s p$ & 197 & hcp & \\
\hline & ds & 213 & bcc & & $\mathrm{Ru}$ & $d(7) s$ & 0 & - & \#\# \\
\hline \multirow[t]{3}{*}{ Sc } & $\mathrm{ds}(2)$ & 0 & - & & & $d(6) s p$ & 301 & hcp & \\
\hline & $\mathrm{dsp}$ & 188 & hcp & & $\mathrm{Rh}$ & $d(8) s$ & 0 & - & \\
\hline & $d(2) s$ & 138 & bcc & & & $\mathrm{d}(6) \operatorname{sp}(2)$ & 536 & fcc & \\
\hline \multirow[t]{3}{*}{$\mathrm{Ti}$} & $\mathrm{d}(2) \mathrm{s}(2)$ & 0 & - & & $\mathrm{Pd}$ & $d(10)$ & 0 & - & \\
\hline & $\mathrm{d}(2) \mathrm{sp}$ & 188 & hcp & & & $\mathrm{d}(7) \mathrm{sp}(2)$ & 586 & fcc & \\
\hline & $\mathrm{d}(3) \mathrm{s}$ & 79 & bcc & & $\mathrm{Ag}$ & $d(10) s$ & 0 & - & \\
\hline \multirow[t]{3}{*}{ V } & $d(3) s(2)$ & 0 & - & & & $\mathrm{d}(8) \operatorname{sp}(2)$ & 556 & fcc & $\#$ \\
\hline & $\mathrm{d}(3) \mathrm{sp}$ & 196 & - & & $\mathrm{Cd}$ & $d(10) s(2)$ & 0 & - & \\
\hline & $d(4) s$ & 25 & bcc & & & $\mathrm{d}(8) \mathrm{sp}$ & 364 & hcp & \\
\hline \multirow[t]{3}{*}{$\mathrm{Cr}$} & $d(5) s$ & 0 & bcc & & $\mathrm{Ba}$ & $s(2)$ & 0 & - & \\
\hline & $d(4) s p$ & 297 & - & & & ds & 109 & bcc & $\#$ \\
\hline & $\mathrm{d}(4) \operatorname{sp}(2)$ & 690 & - & & & $\mathrm{sp}$ & 146 & - & \\
\hline \multirow[t]{3}{*}{ Mn } & $d(5) s(2)$ & 0 & - & & $\mathrm{La}$ & $\mathrm{ds}(2)$ & 0 & - & \\
\hline & $d(5) s p$ & 221 & - & $\#$ & & dsp & 159 & hcp & \\
\hline & $d(6) s$ & 200 & - & & & $d(2) s$ & 33 & bcc & \\
\hline \multirow[t]{4}{*}{$\mathrm{Fe}$} & $\mathrm{d}(6) \mathrm{s}(2)$ & 0 & - & & & $\mathrm{sp}(2)$ & 184 & fcc & \\
\hline & $d(6) s p$ & 230 & - & & $\mathrm{Hf}$ & $\mathrm{d}(2) \mathrm{s}(2)$ & 0 & - & \\
\hline & $d(7) s$ & 83 & bcc & $\#$ & & $\mathrm{~d}(3) \mathrm{s}$ & 167 & bcc & \\
\hline & $\mathrm{d}(5) \operatorname{sp}(2)$ & 384 & $\mathrm{fcc}$ & & & $d(2) s p$ & 213 & hcp & \\
\hline \multirow[t]{4}{*}{ Co } & $d(7) s(2)$ & 0 & - & & $\mathrm{Ta}$ & $d(3) s(2)$ & 0 & - & \\
\hline & $\mathrm{d}(6) \operatorname{sp}(2)$ & 498 & $\mathrm{fcc}$ & & & $\mathrm{d}(4) \mathrm{s}$ & 117 & bcc & \\
\hline & $d(7) s p$ & 280 & hcp & $\#$ & W & $d(4) s(2)$ & 0 & - & \\
\hline & $d(8) s$ & 42 & - & & & $\mathrm{d}(5) \mathrm{s}$ & 33 & bcc & \\
\hline \multirow[t]{4}{*}{$\mathrm{Ni}$} & $d(8) s(2)$ & 0 & - & & $\operatorname{Re}$ & $d(5) s(2)$ & 0 & - & \\
\hline & $d(9) s$ & 4 & - & $\#$ & & $d(6) s$ & 142 & - & \\
\hline & $\mathrm{d}(8) \mathrm{sp}$ & 310 & - & & & $\mathrm{d}(5) \mathrm{sp}$ & 226 & hcp & \\
\hline & $d(7) \operatorname{sp}(2)$ & 502 & $\mathrm{fcc}$ & & Os & $d(6) s(2)$ & 0 & - & \\
\hline \multirow[t]{2}{*}{$\mathrm{Cu}$} & $d(10) s$ & 0 & - & & & $\mathrm{d}(7) \mathrm{s}$ & 63 & - & \\
\hline & $\mathrm{d}(8) \operatorname{sp}(2)$ & 502 & fcc & $\#$ & & $d(6) s p$ & 280 & hcp & \\
\hline \multirow[t]{2}{*}{$\mathrm{Zn}$} & $d(10) s(2)$ & 0 & - & & Ir & $d(7) s(2)$ & 0 & - & \\
\hline & $\mathrm{d}(10) \mathrm{sp}$ & 389 & hcp & & & $d(7) s p$ & 314 & - & \\
\hline \multirow[t]{3}{*}{$\mathrm{Sr}$} & $s(2)$ & 0 & - & & & $\mathrm{d}(6) \operatorname{sp}(2)$ & 678 & fcc & \\
\hline & ds & 217 & bcc & * & $\mathrm{Pt}$ & $d(9) s$ & 0 & - & \\
\hline & $\mathrm{sp}$ & 171 & - & & & $\mathrm{d}(7) \operatorname{sp}(2)$ & 653 & fcc & \\
\hline \multirow[t]{3}{*}{$\mathrm{Y}$} & $\mathrm{ds}(2)$ & 0 & - & & $\mathrm{Au}$ & $\mathrm{d}(10) \mathrm{s}$ & 0 & - & $\#$ \\
\hline & $d(2) s$ & 130 & bcc & & & $\mathrm{d}(8) \operatorname{sp}(2)$ & 665 & fcc & \\
\hline & dsp & 180 & hcp & & $\mathrm{Hg}$ & $d(10) s(2)$ & 0 & - & \\
\hline $\mathrm{Zr}$ & $\mathrm{d}(2) \mathrm{s}(2)$ & 0 & - & & & $d(10) s p$ & 444 & - & \#\#\# \\
\hline
\end{tabular}

(i) The principal quantum numbers are omitted for clarity.

(ii) The number inside the paranthesis stands for the number of electrons in the particular sub shell.

(iii) All promotion energy values are from Brewer ${ }^{(4)}$; lanthanides and actinides are not discussed here, but may be obtained from Ref. (16).

(iv) *: denotes wrong structure assignment as per EBECM.

(v) \#: denotes the case where d-electron count is neglected.

(vi) \#\#: refer text for $\mathrm{Fe}, \mathrm{Ru}$ anamoly.

(vii) \#\#\#: Distorted (close packed) structure; refer text. 
that they do not decide the choice of the crystal structure. This point remains rather unclarified in the frame work of Engel's initial proposition ${ }^{(3)(9)}$, and is therefore a subject of intense debate ${ }^{(2)}$. A general consideration of EBECM leads to the following rules:

(i) The crystal structure depends only on the total number of unpaired $s$ and $p$ electrons. The crystal structures and the respective numbers of $s+p$ unpaired electrons are given as under:

Body Centered Cubic (bcc): 1 electron

Hexagonal Close Packing (hcp): 2 electrons

Face Centered Cubic (fcc): 3 electrons

Diamond Cubic (dc): 4 electrons

(ii) These integral numbers for metallic valence were later modified to cover a range of fractional valencies to account for each crystal structure encountered in certain binary phase fields. In general the following range of values were assigned based on an analysis of binary phase fields.

$$
\begin{array}{ll}
\text { bcc: } & 1.0-1.5 \\
\text { hcp: } & 1.7-2.1 \\
\text { fcc: } & 2.5-3.0
\end{array}
$$

In Table 1, we summarise the data on the ground state and the Brewer's metallic state electron configurations ${ }^{(4)(16)}$ for many metals except lanthanides and actinides for which it may be obtained from reference ${ }^{(16)}$.

\section{Limitations of Engel-Brewer model}

The direct non incorporation of d-electrons for transition metals in the crystal structure-valence electron configuration correlation is an obvious point for criticism. As mentioned earlier the Engel-Brewer model allows for $\mathrm{d}-\mathrm{s} / \mathrm{p}$ hybridization in explaining transition metal cohesion. However it is rather mystifying to think that, d-electrons while contributing to crystal cohesion, do not play a role in deciding the crystal structure. It appears that according to EBECM the crystal structure vis-a-vis the chemical binding is decided by entirely different set of electrons. It is interesting to note that the metallic state as portrayed by condensed matter physics offers a conflicting picture ${ }^{(1)(2)(12)-(15)(17)-(19)}$. The work of Pettifor $^{(18)(19)}$ among others ${ }^{(20)}$ strongly suggests that for transition metals the d-electrons contribute significantly towards cohesion and further determine the structure as well. Of course the subsidiary contributions arising from $\mathrm{d}-\mathrm{s} / \mathrm{p}$ mixing etc. do play a non negligible role $\mathrm{e}^{(13)(14)(17)(20)}$. These finer aspects do not concern us here. In brief, it may be said that viewpoints based on band theoretical considerations are at variance with that of EngelBrewer's regarding structural stability.

For transition metals it may be noticed from Table 1, that for the first two elements of each $3 \mathrm{~d}, 4 \mathrm{~d}$ and $5 \mathrm{~d}$ long periods, the promotion energies for $\mathrm{s}$ and sp configurations are rather close enough to probably justify the presence of both bcc and hcp structures. The bcc structure at high temperature is attributed to entropic considerations; but this must be weighed together with competing interactions. At high temperatures there may be phase changes occurring when electrons near the Fermi level are promoted to non bonding states ${ }^{(15)}$. Brewer proposed that for refractory metals like $\mathrm{V}, \mathrm{Nb}$ and $\mathrm{Ta}$, the promotion energies for $\mathrm{d}^{4} \mathrm{~s}$ configuration are small enough to permit bcc as the stable structure. The noteworthy point here is the total neglect of d-electrons in deciding the structure.

The case of manganese raises another interesting point. The energy cost for manganese to have a $d^{5} s p$ promoted state is about $222 \mathrm{~kJ} / \mathrm{mol}$ and in comparison with other promotion energy values (Table 1), it seems probable that a hcp structure for manganese can exist. The observed fcc structure on the contrary necessitates a $\mathrm{d}^{4} \mathrm{sp}^{2}$ valence state as per Engel-Brewer scheme. This is rather difficult to reconcile with, since the removal of a delectron from half-filled $\mathrm{d}^{5}$ state would cost enormously. The physical reasoning for the existence of manganese structure must therefore be sought elsewhere.

Iron and ruthenium pose another set of interesting examples: for iron, an hcp structure appropriate to the $d^{6} \mathrm{sp}$ state does not occur in the ambient conditions while for ruthenium, the listed $\mathrm{d}^{7} \mathrm{~s}$ configuration yields as per EBECM only bcc as the possible structure. But ruthenium with bcc modification does not exist at all. In this context it is interesting to note that the promotion energy for ruthenium to have a $\mathrm{d}^{6} \mathrm{sp}$ excited state consistent with its hcp crystal structure is high $(301 \mathrm{~kJ} / \mathrm{mol})$; in fact it is even higher than that for iron $(222 \mathrm{~kJ} / \mathrm{mol})$. Yet ruthenium exists only in hcp form at all temperatures, whereas for iron high pressure conditions are needed to induce a structural transformation from bcc to hcp. The difference in the qualitative behaviour of iron and ruthenium calls for its understanding, a direct consideration of delectrons in explaining cohesion and structure. Besides these examples it has already been mentioned that the promotion energy required for the postulated $d^{8} \mathrm{sp}^{2}$ state with three $(s+p)$ valence electrons in the case of noble metals is rather high to favour the fcc structure. The contribution of d-electrons to bonding charge density in case of copper is now established ${ }^{(21)}$ and here again a definite influence of d-band must be taken in to account. In other words the association of $\mathrm{sp}^{2}$ with fcc must be abandoned and in its place a new correlation involving d-electron counts must be substituted.

Apart from this limitation for transition metals, the EBECM also fails for some alkaline earth metals. Thus for calcium and strontium, we have only two valence electrons (Table 1); but the observed fcc structure as per EBECM requires three electrons. Secondly, for noble metals, the E-B scheme allocates a $\mathrm{d}^{8} \mathrm{sp}^{2}$ configuration with rather high promotion energy values (Table 1). If the actual metallic configuration is $\mathrm{d}^{8} \mathrm{sp}^{2}$, then this is inconsistent with the observed diamagnetism of the noble metals in general ${ }^{(15)}$. Further there seems to be no obvious physical reasoning for the occurrence of slightly distorted versions of close packed structures adopted by say gallium, in terms of the basic tenets of the E-B theory. Lithium and sodium have hcp structures at low temperatures and this too cannot be accounted for by the E-B scheme. To invoke an hcp structure for these metals with one $s$ 
electron in the outer shell, one has to promote this electron to the next $\mathrm{p}$ shell which would involve a large amount of excitation energy.

Having seen the limitations in general, it must be mentioned to its credit, that the Engel-Brewer theory paved way for the adroit use of promoted energy levels in tackling issues related with structural stability ${ }^{(22)}$. This is because the chemical basis of EBECM is simple and transparent, but not transferable enough. Therefore EngelBrewer rules, being classical in origin and content, need to be modified to accomodate the current viewpoints regarding crystal structure trends. Towards this goal, we shall briefly review the current understanding on structural stability of metals in the ensuing section.

\section{Cohesion and Structural Stability: Physical Viewpoints}

In this section let us review the key concepts prevalent in solid state physics in an axiomatic manner to enable the qualitative picture of metallic cohesion be grasped rather easily. A detailed account of the recent developments may be obtained from the monographs of Hafner $^{(12)}$ and Ducastelle ${ }^{(13)}$ for $\mathrm{s} / \mathrm{p}$ metals-alloys and transition metals-alloys respectively. Also the book by Cottrell $^{(14)}$ presents lucidly the modern theory of metals.

\section{Non-transition metals}

Most of the simple or non transition metals have long been pictured as consisting of positive ion cores immersed in a sea of nearly free electrons. In this case a significant amount of the electrostatic electron-ion interaction is offset by the repulsive forces generated by high kinetic energy valence electron interactions ${ }^{(12)}$. This enables one to define a weak 'pseudopotential' for the outer electrons moving in a periodic lattice of normal metals. Within the frame work of pseudopotential concept, the total energy is written to second order as ${ }^{(12)}$

$$
E=F(\Omega)+\frac{1}{2} \sum_{R \neq 0} \Phi(R)
$$

where $F(\Omega)$ is a function of atomic volume $(\Omega)$, consisting of kinetic, exchange and correlation contributions of a nearly homogeneous electron gas. The second term in eq. (1) is the pairwise interaction term, whose general form again using simple model pseudo potentials may be inferred as:

$$
\begin{aligned}
& \Phi(R)=\Phi_{r e d}(R)\left(Z^{2} / R\right) \\
& \Phi_{r e d}(R)=\left[(2 R) /\left(\pi \epsilon^{2}\left(2 k_{\mathrm{F}}\right)\right)\right]\left[\cos \left(2 k_{\mathrm{F}} R\right) /\left(2 \pi k_{\mathrm{F}} R\right)^{3}\right]
\end{aligned}
$$

in eq. (2) $Z$ is the metallic valence, $R$ is the distance coordinate, $k_{\mathrm{F}}$ is the Fermi wave number, and $\epsilon$ is the energy of the electron corresponding to the Fermi level. All these quantities can be readily calculated in terms of the nearly free electron model. Our objective in invoking eq. (2) is that, since for normal metals the crystal structure trends may be interpreted by the actual form of $\Phi(R)$ so that, $\Phi(R)$ when normalized with respect to $Z$ is principle capable of explaining the crystal structure trends across the periodic table. Equations (1) and (2) in fact encompass a series of assumptions, which however are of no concern for our qualitative understanding in the present context. In Fig. 1 we present a schematic of $\Phi(R)$ vs. $R$ curve for a typical metal like gallium. The reason for taking gallium as an example will become obvious soon. As is evident from eq. (2) and from Fig. $1, \Phi(R)$ is an oscillating function with the wave length of oscillations being determined by $k_{\mathrm{F}}$. In Fig. 1 the actual minimum in the curve does not coincide with the true near neighbour distance of the crystal lattice. This is not conflicting since the equilibrium spacing is determined by minimising $E$ the total electronic ground state energy, while the minimum in the $\Phi(R)$ versus $R$ curve (Fig. 1) is decided by the amplitude factor in eq. (2). However, any drastic deviation of these two characteristic points is not allowed as it would cost too much in terms of energy ${ }^{(12)}$. The crucial point is that if the nearest neighbour distance of a particular crystal lattice happens to fall near the peak in the potential versus distance curve as it occurs for example in the case of gallium (Fig. 1; where a close packed structure is assumed), the structure is unstable and can lower its energy by a rearrangement of its first shell neighbours in such a manner that some of them are brought closer while the others pulled further away from the predicted minimum in the $\Phi(R)$ versus $R$ curve. As per pseudopotential arguments this is the physical reason for the occurrence of distorted close packed structure in gallium. It must also be added that for most of the close packed normal metals, the minimum in $\Phi(R)$ nearly coincides with the equilibrium near neighbour spacing and explaining therefore the stability of close packed structures for these simple metals. When going to covalent structures like that of silicon for example, the interatomic potential at the equilibrium near neighbour spacing is such that both the first and second derivatives of the potential

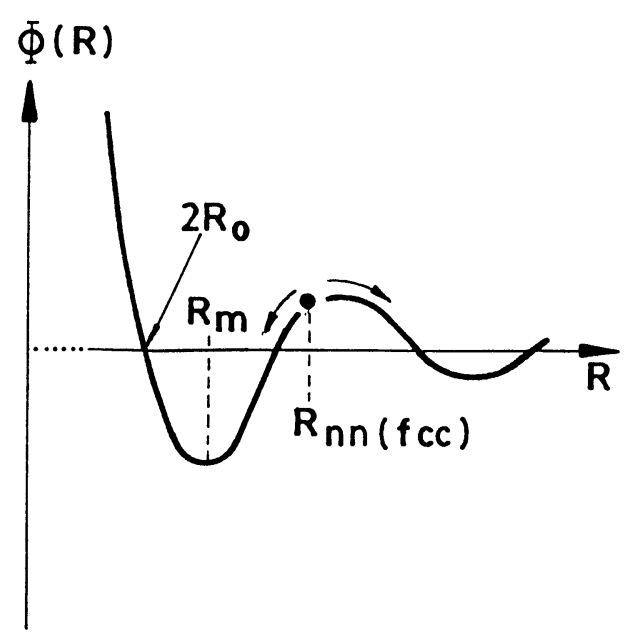

Fig. 1 A schematic pairwise interatomic potential curve for metals like gallium based on pseudopotential considerations. Note that the minimum in the curve does not coincide with the equilibrium spacing of the gallium lattice (refer text for details). 
(with respect to $R$ ) are negative, indicating clearly the instability of a close packed structure. Stating without elaboration, the presence of d-character in the bonding region for many B-subgroup elements as well as their group V counterparts, results in an increase in valence charge density. This in turn shifts the minimum point in Fig. 1 away from the nearest neighbour spacing of a corresponding close packed structure making thus the distorted versions of close packed arrangements possible for these elements. Finally when the d-component contribution becomes appreciable as in the case of transition metals, the simple pseudopotential picture breaks down. Summarising the crystal structure trends of nontransition metals, we may conclude by saying that there seems to be no compelling support from solid state theory for the sole $n_{\mathrm{s}+\mathrm{p}}$ dependence of crystal structure. As per eqs. (1) and (2) the $Z^{2} / R$ term becomes dominant as $R \rightarrow \propto$; in other words this electrostatic term standing for the effective potential seen by the valence electron at an appropriate radius $R$, if considered alone (structural stability requires of course a repulsive term as well, which can be a simple Born type for our purposes here) would favour compact crystal structures. The presence of cosine terms representing the oscillatory behaviour is the one which determines the relative structural stability. Elaborate arguments within the framework of pseudopotential theory on crystal structure trends are presented by Hafner ${ }^{(12)}$ and the interested readers are referred to his monograph for further details in this direction.

\section{Transition metals}

Table 2 presents the observed crystal structure sequence in case of $3 \mathrm{~d}, 4 \mathrm{~d}$ and $5 \mathrm{~d}$ transition metals. Here the close packed structures are preferred at either end of the series while bcc is favoured in the middle. In $3 \mathrm{~d}$ the magnetic effects are important and play a role in cohesion and structure ${ }^{(13)}$. Barring the magnetic effect, a one electron based description of electronic structure provides a satisfactory description for many a solid state phenomenon. At the outset it may be said in general terms that the band structure of transition metals consists of a narrow d-band with a large density of states near the Fermi level and a broad band derived from $s$ and $p$ states. It is now established that d-electrons in transition metals are itinerant (contributing to transport properties) while also accounting for their magnetic properties. But unlike the $s$ and $\mathrm{p}$ bands, this d-band contribution cannot be ex-

Table 2 Structure of d-transition metals. Note that the close packed structures are favoured at either end of the series, while bcc is preferred at the middle. The structure of $\mathrm{La}$ is dhcp, but is categorized under hcp for simplicity. Similarly the actual structure of $\mathrm{Mn}$ is complex, but is again included under hcp. The bcc structure of Fe is due to magnetic considerations.

\begin{tabular}{|c|c|c|c|}
\hline hcp & bcc & hcp & fcc \\
\hline $\mathrm{Sc} \quad \mathrm{Ti}$ & $\mathrm{V} \mathrm{Cr} \mathrm{Fe}$ & $\mathrm{Mn}$ Co & $\mathrm{Ni} \mathrm{Cu}$ \\
\hline $\mathrm{Y} \quad \mathrm{Zr}$ & $\mathrm{Nb} \quad \mathrm{Mo}$ & $\mathrm{Tc} \mathrm{Ru}$ & $\mathrm{Rh} \quad \mathrm{Pd} \quad \mathrm{Ag}$ \\
\hline $\mathrm{La} \quad \mathrm{Hf}$ & $\mathrm{Ta} \mathrm{W}$ & $\mathrm{Re} O \mathrm{Os}$ & Ir $\mathrm{Pt} \quad \mathrm{Au}$ \\
\hline
\end{tabular}

pressed in terms of model pairwise interactions ${ }^{(13)(23)}$. At present cohesion in transition metals and their alloys is understood within the framework of what is known as the 'Tight Binding Model', in which to a first order of approximation the cohesive energy turns out to be independent of structure. Actually the relative structural stability comes from small differences in band structure contribution to total electronic energy and whose adequate description calls for the inclusion higher order moments in describing the density of states curve. There is no room here for a detailed discussion of this topic and for which, we refer to the most comprehensive monograph of Ducastelle ${ }^{(13)}$. Stating rather phenomenologically the binding energy of a transition metal may be written as a summation of following contributions:

$$
E=E_{\mathrm{d}}+E_{\mathrm{s}, \mathrm{p}}+E_{\mathrm{s}-\mathrm{d} \text { mixing }}+E_{\text {others }}
$$

of these, $E_{\mathrm{d}}$ represents the contribution from d-band broadening and this is normally a dominant contributor. $E_{\mathrm{s}, \mathrm{p}}$ stands for the $\mathrm{s}$ and $\mathrm{p}$ contribution to cohesion and $E_{\mathrm{s}-\mathrm{d} \text { mixing }}$ denotes the hybridization contribution. The last term in eq. (3) includes contributions from dynamical correlation effects which are of importance in explaining magnetic effects.

Barring noble metals (where the hybridization term makes non negligible contribution in eq. (3)) and the magnetic ones, the trends in cohesive energy may be successfully explained in terms of the $E_{\mathrm{d}}$ term using a simple rectangular model density of states for the d-band. As originally proposed by Friedel this may be given as

$$
E_{\mathrm{d}}=-\left(Z_{\mathrm{d}} / 20\right)\left(10-Z_{\mathrm{d}}\right) W_{\mathrm{d}}
$$

where $W_{\mathrm{d}}$ represents the width of the d-band and $Z_{\mathrm{d}}$ the number of bonding d-electrons. It is evident from eq. (4) that the maximum in cohesive energy is expected at the middle of the series which is in agreement with the experimental results. Pettifor ${ }^{(24)}$ has shown that the structural choice among transition metals exhibits a regularity with $Z_{\mathrm{d}}$, the bonding d-electron number. This is illustrated in Fig. 2, wherein the relative structural stability is delineated against $Z_{\mathrm{d}}$. The presence of bcc structure for the half-filled or nearly half-filled state comes from the greater separation of bonding and antibonding states with bonding states being nearly filled ${ }^{(24)}$. The structural difference between hcp and fcc oscillates around zero energy and this may be reconciled to a first approximation in terms of tight binding model, since in both fcc and hcp the near neighbour distances may be considered to be the same under constant volume enabling thus the transfer integrals to have identical values for these two structures. The transfer integral is a measure of energy required for the transfer of electrons between near neighbour sites and in a loose sense identifies an effective ionic contribution. It may be mentioned in this context that the role of further neighbour interactions assumes a non trivial importance. Of course higher order moments are to be necessarily invoked for a satisfactory explanation of the bcc phase around the middle of transition series. 


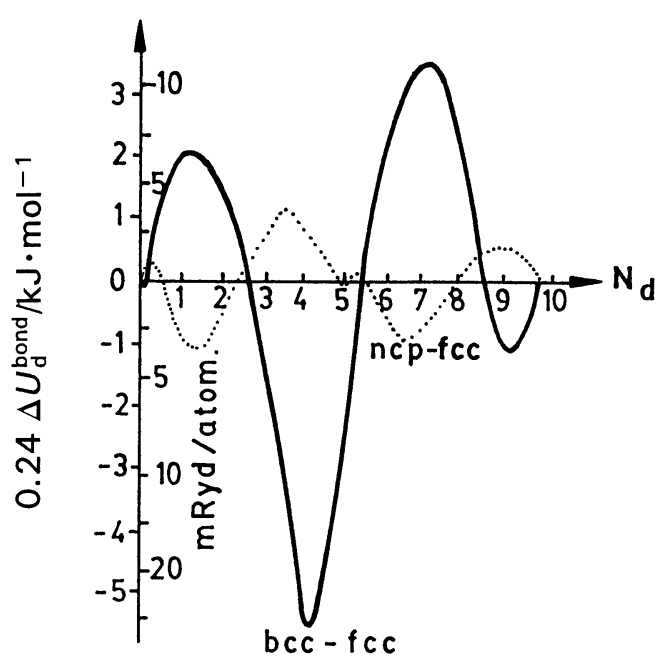

Fig. 2 The relative stability of hcp, fcc and bcc structures in the case of transition metals, as obtained through the tight binding scheme is schematically illustrated.

We may conclude this section by saying that the number of d-electrons exerts a definite influence in the crystal structure choice for reasons that are altogether different from what Brewer has alluded to. Further that the d-electrons do not serve to determine the crystal structure is not supported by d-band physics. In fact the number of $s+p$ electrons in many of the model density of states studies for transition metals is found to be roughly constant through out the series (about 1-2 on the average). Only the number of d-electrons is found to vary. If $s$ and $p$ electrons alone were to determine the structure as per Brewer, we would end up with a single structure for all the transition metals. Further it has been shown that structure maps involving d-band coordinates are eminently successful in sorting various structures of transition metal compounds ${ }^{(25)}$. This, viewed with the basic success of tight binding picture of transition metals and their alloys point decisively towards the technical inexactitude of classical Engel-Brewer scheme. Despite remarkably successful in those cases where it is so, the success should not be taken to imply the correctness of the alloying theory.

In the following section we modify the original Brewer's configuration for transition metals, some alkaline earths and noble metals by substituting instead a valence configuration which takes in to account directly the d-electrons.

\section{Modified Valence Electron Model}

The revised metallic state electron configurations are presented in Table 3. In general we propose a $\mathrm{d}^{n-x} \mathrm{sp}^{x}$ state, allowing thereby explicitly the s-d hybridization. If the d-level energy is low then the bonding is controlled mostly by the $s+p$ electrons, which are now nearer to the Fermi level. If on the other hand the d-level energy is high, we would expect them to be nearer to the Fermi level and contribute significantly to structure, cohesion and
Table 3 Revised valence configuration for alkali, alkaline earth and d-transition metals.

\begin{tabular}{|c|c|c|c|c|}
\hline \multirow[b]{2}{*}{ Element } & \multirow{2}{*}{$\begin{array}{c}\text { Revised } \\
\text { valence state } \\
\text { configuration }\end{array}$} & \multicolumn{2}{|c|}{ Valence configurations } & \multirow[b]{2}{*}{ Remarks } \\
\hline & & $\begin{array}{l}\text { Niemienen \& } \\
\text { Hodges }\end{array}$ & $\begin{array}{l}\text { Lang \& } \\
\text { Williams }\end{array}$ & \\
\hline $\mathrm{Na}$ & $s(1)$ & - & - & \\
\hline $\mathrm{Mg}$ & $\mathrm{s}(2-x) \mathrm{p}(x)$ & - & - & $x=0.1$ \\
\hline $\mathrm{Al}$ & $s(2) p$ & - & - & \\
\hline $\mathbf{K}$ & $\mathrm{d}(0) \mathrm{s}(1)$ & - & - & \\
\hline \multirow[t]{2}{*}{$\mathrm{Ca}$} & $\mathrm{d}(x)(\mathrm{s}, \mathrm{p})$ & & & \\
\hline & $(1-x)$ & - & $3 \mathrm{~d}(0.5)$ & $x=0.5$ \\
\hline $\mathrm{Sc}$ & $\begin{array}{l}\mathrm{d}(2-x), \\
(\mathrm{s}, \mathrm{p})(x)\end{array}$ & $3 \mathrm{~d}(1.8)$ & $3 \mathrm{~d}(1.6)$ & $x=0.2-0.4$ \\
\hline \multirow[t]{2}{*}{$\mathrm{Ti}$} & $\mathrm{d}(3-x)$ & & & \\
\hline & $\begin{array}{l}(\mathrm{s}, \mathrm{p})(x) \\
\mathrm{d}(4-x)\end{array}$ & $3 \mathrm{~d}(2.7)$ & $3 \mathrm{~d}(2.7)$ & $x=0.3$ \\
\hline V & $\mathrm{s}(1+x)$ & $3 \mathrm{~d}(3.9)$ & $3 \mathrm{~d}(3.7)$ & $x=0.1-0.2$ \\
\hline \multirow[t]{2}{*}{$\mathrm{Cr}$} & $\mathrm{d}(5-x)$ & & & \\
\hline & $\mathrm{s}(1+x)$ & $3 \mathrm{~d}(4.7)$ & $3 \mathrm{~d}(4.6)$ & $x=0.3-0.4$ \\
\hline $\mathrm{Mn}$ & $\begin{array}{l}\mathrm{d}(6-x) \\
\mathrm{sp}(x)\end{array}$ & $\begin{array}{c}3 \mathrm{~d}(5.6) \\
\text { interpolated }\end{array}$ & $3 d(5.6)$ & $\begin{array}{c}x=0.4-0.5 \\
\text { tentative }\end{array}$ \\
\hline $\mathrm{Fe}$ & $\begin{array}{l}\mathrm{d}(7-x) \\
(\mathrm{s}, \mathrm{p})(x)\end{array}$ & $3 d(6.6)$ & $3 \mathrm{~d}(6.6)$ & $\begin{array}{c}x=0.4 \\
\text { tentative }\end{array}$ \\
\hline Co & $\begin{array}{l}\mathrm{d}(8-x) \\
\mathrm{s}, \mathrm{p}(x) \\
\mathrm{d}(9-x)\end{array}$ & $3 \mathrm{~d}(7.6)$ & $3 \mathrm{~d}(7.6)$ & $x=0.4$ \\
\hline $\mathrm{Ni}$ & $\begin{array}{l}\mathrm{s}, \mathrm{p}(x) \\
\mathrm{d}(10-x)\end{array}$ & $3 \mathrm{~d}(8.7)$ & $3 d(8.6)$ & $x=0.4-0.3$ \\
\hline $\mathrm{Cu}$ & $\mathrm{s}, \mathrm{p}(\mathrm{x})$ & $3 \mathrm{~d}(9.7)$ & $3 \mathrm{~d}(9.6)$ & $x=0.4-0.3$ \\
\hline $\mathrm{Rb}$ & $s(1)$ & - & - & \\
\hline $\mathrm{Sr}$ & $\mathrm{d}(x) \operatorname{sp}(1-x)$ & - & - & $x=0.5$ \\
\hline $\mathbf{Y}$ & $\mathrm{d}(2-x) \operatorname{sp}(x)$ & $\mathrm{d}(1.5)$ & - & $x=0.5$ \\
\hline $\mathrm{Zr}$ & $\mathrm{d}(3-x) \operatorname{sp}(x)$ & $d(2.7)$ & - & $x=0.3$ \\
\hline $\mathrm{Nb}$ & $d(4) s(1)$ & $\mathrm{d}(4)$ & - & \\
\hline Mo & $\mathrm{d}(5-x) \mathrm{s}(1+x)$ & $\mathrm{d}(4.8)$ & - & $x=0.2$ \\
\hline $\mathrm{Tc}$ & $\mathrm{d}(6-x) \operatorname{sp}(x)$ & - & - & $x=0.5$ \\
\hline $\mathrm{Ru}$ & $\mathrm{d}(7-x) \operatorname{sp}(x)$ & - & - & $x=0.4$ \\
\hline $\mathrm{Rh}$ & $\mathrm{d}(8-x) \mathrm{s}$ & $\mathrm{d}(7.7)$ & - & $x=0.3$ \\
\hline $\mathrm{Pd}$ & $d(9) s$ & $\mathrm{~d}(9.1)$ & - & $*$ \\
\hline $\mathrm{Ag}$ & $\mathrm{d}(10-x) \operatorname{sp}(x)$ & $\mathrm{d}(9.7)$ & - & $x=0.3$ \\
\hline Cs & $s(1)$ & - & - & \\
\hline $\mathrm{Ba}$ & $\mathrm{d}(x) \operatorname{sp}(1-x)$ & & & $x=0.5$ \\
\hline $\mathrm{La}$ & $\mathrm{d}(2-x) \operatorname{sp}(x)$ & - & - & $x=0.5$ \\
\hline $\mathrm{Hf}$ & $\mathrm{d}(3-x) \operatorname{sp}(x)$ & - & - & \\
\hline $\mathrm{Ta}$ & $\mathrm{d}(4-x) \operatorname{sp}(x)$ & $d(4)$ & - & $x=0-0.5$ \\
\hline W & $d(5) s$ & $d(4.7)$ & - & \\
\hline $\operatorname{Re}$ & $\mathrm{d}(6-x) \operatorname{sp}(x)$ & - & - & \\
\hline Os & $d(7) s$ & - & - & \\
\hline Ir & $\mathrm{d}(8) \mathrm{s}$ & $d(7)$ & - & \\
\hline $\mathrm{Pt}$ & $d(9) s$ & - & - & $*$ \\
\hline $\mathrm{Au}$ & $\mathrm{d}(10-x) \operatorname{sp}(x)$ & $\mathrm{d}(8.8)$ & - & $x=1.2$ \\
\hline
\end{tabular}

${ }^{*}$ Note that for $\mathrm{Pd}$ and $\mathrm{Pt}, \mathrm{a} \mathrm{d}(9+x)$ with small $x$ is appropriate; Nieminen-Hodges ${ }^{(26)}$ configuration suggests that the direction of (intra) electron transfer in these two cases is different from that of in nickel. In general the $x$ values given here are only suggestive; a better estimate can however be made by studying the available bonding charge density distribution.

metallic properties. It may be said in general terms that $d$ band contribution enhances as one goes down the group.

In Table 3, we also list the valence configurations due to Nieminen and Hodges ${ }^{(26)}$, Lang and Williams ${ }^{(27)}$. We use these auxiliary data to determine the approximate 
values of $x$ in our empirically proposed configurations. In the light of the new configuration the correlation with crystal structure is as follows:

(i) For metals with $d^{0}-d^{2}$, we assign fcc structure, while for $\mathrm{d}^{2}-\mathrm{d}^{4}$ we assign hcp,

(ii) For metals with a near $\mathrm{d}^{5}$ configuration, that is half filled $d$ shell we assign bcc structure,

(iii) While calculating the effective or bonding d-electrons,

we assume that $Z_{\mathrm{d}}=n_{\mathrm{d}}$ or $10-n_{\mathrm{d}}$ depending on whether $n_{\mathrm{d}}$ is less than or equal to five or greater than five respectively. $Z_{\mathrm{d}}$ is the number of effective bonding d-electrons and $n_{\mathrm{d}}$ is the total d-electron count.

Let us briefly review the extended electron correlation model for its utility in systematizing the crystal structure of metallic elements. For alkali metals the $\mathrm{d}^{0} \mathrm{~s}^{1}$ configuration as originally suggested by Brewer is retained. For alkaline earth metals, the d-orbital energies are near Fermi level ${ }^{(28)}$ and hence there is a possibility of enhanced s-d hybridization as we go down the group. We accordingly suggest $\mathrm{d}^{x} \mathrm{sp}^{1-x}$ configurations with $x \approx 0.5$. The presence of fcc structure in this case must directly arise from d-electrons. The pressure induced transformations also support this conclusion. Thus the heavy alkalis, $\mathrm{K}$, $\mathrm{Rb}$ and $\mathrm{Cs}$ transform from bcc to fcc and the alkaline earths $\mathrm{Ca}$ and $\mathrm{Sr}$ transform from fcc to bcc (at low temperatures) ${ }^{(12)}$. It has been demonstrated that these phase changes are closely related to the changes in d-band occupancy under external influence; that is under pressure the s-levels are shifted upwards and the d-levels are pushed downwards enabling thus an enhancement of $s$ to $\mathrm{d}$ electron transfer. It may be said that under high pressure conditions heavy alkalis and alkaline earths behave like d-transition metals. Recently the d-band driven pressure induced transformations are suggested even for $\mathrm{Na}$, $\mathrm{Mg}$ and $\mathrm{Al}^{(12)}$. By proposing a $\mathrm{d}^{x} \mathrm{sp}^{1-x}$ valence state with variable $\mathrm{x}$ depending on the experimental conditions, most of these structural changes are qualitatively accounted for.

Coming to transition metals, their structure as correlated to the number of d-electrons is amply borne out by our modified valence configuration. Only for manganese and iron the correlation runs in to troubles. It is known that magnetic effects are important in $3 \mathrm{~d}$ and that it might stabilise the bcc structure of iron. There is a viewpoint that $\alpha$-Mn structure may be stabilised because of crystal field effects. In either case an one electron based picture cannot clearly explain the stability and our proposed configuration in this case bears no improvement over Brewer's. Further Brewer attributes the presence of many allotropes for manganese (and plutonium as well) to the possibility of many excited states with small energy differences and that some of the atoms may reside permanently in the excited state ${ }^{(4)}$. This hypothesis remains yet to be proven experimentally. Barring these 3d exceptions, as we go to Tc and Re the stability of hcp is correctly restored as per $\mathrm{d}^{6-x} \mathrm{sp}^{x}$ state.

For noble metals the presence of d-electrons in the valence region is well documented ${ }^{(15)}$. Therefore it is not unreasonable to expect a $\mathrm{d}^{10-x} \mathrm{sp}^{x}$ configuration with $x$, although small, increasing down the group. For both $\mathrm{Zn}$ and $\mathrm{Cd}$ the outer shells are filled and the promotion from $\mathrm{s}^{2}$ to $\mathrm{s}^{2-x} \mathrm{p}^{x}$ is invoked. The distortion from ideal $\mathrm{c} / \mathrm{a}$ of $\mathrm{hcp}$ is directly explainable in terms of pseudopotential arguments ${ }^{(12)}$.

\section{Discussion}

An appraisal of Engel-Brewer theory from a physics stand point is in fact not new ${ }^{(9)(10)(22)}$. But the philosophical nature of the issues involved makes a definite resolution still not possible. For long it has been recognised that the average electronic configuration in a solid is generally different from the free atom configuration and further that the integral occupation is not a must. It is interesting to recall here an earlier work of Snow and Waber ${ }^{(29)}$ concerning the metallic state electron configuration. They performed (non self consistent) APW calculations for both the bcc and fcc structures of $3 \mathrm{~d}$ transition metals. Using what is known as the orbital decomposition technique applied to crystal orbitals (this involves a projection of each 1-dependent part of the crystal wave function), they found that, not withstanding the limitations of the theoretical methods, the listed d-like population of Brewer is not in agreement with the estimated ones. Moreover it must be added that the recent band structure and density functional studies concerning the stability of alloy phases are definitive in that they do not support in any way the Brewer's hypothesis ${ }^{(13)(14)}$. A modern quantum-chemical picture in terms of valence bond considerations (see Messmer in Ref. (2)) also does not attribute the metallic stability to reasons that can be in consonance with the Brewer's model. The idea that metallic valence is not a ubiquitous bonding parameter is alluded to by Pearson ${ }^{(30)}$ in his book while discussing at length about atom size in solids. It is clear that a single set of radii values cannot serve to systematize the lattice dimensions of solids that differ in their bonding character. However a similar consideration also applies for the e/a concept is not so obvious. The crucial point is that the structure is not only dependent on the number of bonding electrons, but more importantly on their spatial disposition.

The main objective of this paper is to bridge the gap in the classical Engel-Brewer description of bonding in transition metals. In doing so we heavily leaned on the band theoretical considerations of structural stability. We hasten to add that this extension following the premise of EBECM does in no way endorse Brewer's scheme of partitioning the metallic binding. On the other hand it is our hope that it will enhance the applicability as an empirical tool for structural systematisation studies.

\section{Acknowledgements}

The authors acknowledge Dr. Placid Rodriguez for his support and also for providing us with the translated versions of Engel's original papers. They acknowledge Dr. Baldev Raj and Shri. J. B. Gnanamoorthy for their 
encouragement during the course of this work.

\section{REFERENCES}

(1) See for example, J. S. Faulkner: Prog. Mater. Sci., 27 (1982) 1; Highlights in Condensed Matter Theory, ed. by F. Bassani, F. Fumi and M. P. Tosi, North Holland, Amsterdam, (1985).

(2) E. S. Machlin: Metallurgical Treatise, ed. by J. K. Tien and J. F. Elliot, The Met. Soc. AIME, Warrendale, (1981), p. 409; R. P. Messmer, R. C. Tatar and C. L. Briant: Alloying, ed. by J. L. Walter, M. R. Jackson and C. T. Sims, ASM, Metals Park, Ohio, (1988), p. 29.

(3) N. Engel: Kemisk Mandesblaad, 30 (1949), 53, 75, 97, 105 and 114; Powder Met. Bull., 7 (1954), 8; Acta Metall., 15 (1967), 557; Trans. ASM, Quart., 57 (1964), 619.

(4) L. Brewer: Phase Stability in Metals and Alloys ed. by P. S. Rudman, R. I. Jaffee and J. S. Stringer, McGraw Hill, New York, (1967), p. 39, 241, 344 and 560; Alloying, ed. by J. L. Walter, M. R. Jackson and C. T. Sims, Metals Park, Ohio, (1988), p. 1; Pure and Appl. Chem., (IUPAC), 60 (1988), 281.

(5) L. Brewer and R. Lamoreux: Atomic Energy Rev., Spl. Issue No. 7, IAEA, Vienna (1980) p. 1; L. Brewer: J. Mater. Educ., 6 (1984), 733.

(6) L. Brewer: J. Nucl. Mat., 167 (1989), 3.

(7) M. Cima and L. Brewer: Metall. Trans., 19B (1988), 893; L. Brewer: J. Phys. Chem., 94 (1990), 1196 and 7839.

(8) S. L. Altman, C. A. Coulson and W. Hume-Rothery: Proc. Roy. Soc., London., Ser. A, 240 (1957), 145; R. P. Messmer et al.: in Ref. (2).

(9) W. Hume-Rothery: Acta Metall., 13 (1965), 1039 and 15 (1967), 567; Prog. Mater. Sci., 13 (1968), 5; Discussions in High Strength Materials, ed. by V. F. Zackay, John Wiley \& Sons, New York, (1965), p. 70

(10) W. B. Pearson: Developments in the Structural Chemistry of Alloy Phases, ed. by B. C. Giessen, Plenum, New York, (1969), p. 1; see also the discussion note of L. H. Bennett here.

(11) L. Brewer: in Ref. (4); more importantly see the panel discussion by Lomer et al. for a general physical viewpoint of structure vis avis the bonding as prevalent in late sixtees.

(12) J. Hafner: Springer Verlag Series in Solid State Physics, vol. 70,
Springer, Heidelberg, (1990).

(13) F. Ducastelle: Cohesion and Structure series, ed. by F. R. de Boer and D. G. Pettifor, North Holland, Amsterdam, (1991).

(14) A. H. Cottrell: Theory of Metals, Institute of Metals, London, (1991).

(15) P. A. Cox: Electronic Structure and Chemistry of Solids, Oxford University Press, London, (1987).

(16) L. Brewer: Lawrence Berkeley Lab. Report, LBL, 3720, (1975); Heavy Element Properties, ed. by W. Mueller and H. Blank, North Holland, Amsterdam, (1976), p. 30.

(17) A. R. Williams, C. D. Gelatt Jr., J. W. D. Connolly and V. L. Moruzzi: Alloy Phase Diagrams, ed. by L. H. Bennett, T. B. Massalski and B. C. Giessen, MRS Symposium Proceedings, vol. 19, North Holland, Amsterdam, (1983), p. 17.

(18) D. G. Pettifor: J. Phys., C3 (1970), 367, F7 (1977), 613 and F8 (1978), 219.

(19) D. G. Pettifor: Commun. Phys., 1 (1976), 14.

(20) R. E. Watson and L. H. Bennett: Theory of Alloy Phase Formation, ed. by L. H. Bennett, The Met. Soc. AIME, Warrendale, (1980).

(21) R. J. Weiss: Philos. Mag., 26 (1972), 761.

(22) J. T. Waber: Heavy Element Properties, ed. by W. Mueller and H. Blank, North Holland, Amsterdam, (1976), p. 30.

(23) The development of interatomic potential for transition metals is still an active field; consult for example F. Ducastelle in Ref. (13) for details.

(24) D. G. Pettifor: Physical Metallurgy, ed. by R. W. Cahn and P. Haasen, North Holland, Amsterdam, (1982); see also Ref. (18) \& (19).

(25) A. Bieber and F. Gautier: Acta Metall., 34 (1986), 2291; R. E. Watson and L. H. Bennett: Acta Metall., 32 (1984), 491.

(26) R. M. Nieminen and C. H. Hodges: J. Phys., F6 (1976), 573.

(27) N. D. Lang and A. R. Williams: Phys. Rev. Lett., 40 (1978), 954.

(28) P. O. Nilsson, G. Arbman and P. E. Eastman: Solid State Commun., 12 (1969), 627; C. Suguira: Japan J. Appl. Phys., 11 (1972), 598.

(29) E. C. Snow and J. T. Waber: Acta Metall., 17 (1969), 623.

(30) W. B. Pearson: Crystal Chemistry and Physics of Metals and Alloys, Wiley-Interscience, New York, (1972). 